\title{
Quantification of Posterior Risk Related to Intrapartum FIGO 2015 Criteria for Cardiotocography in the Second Stage of Labor
}

\author{
Paolo Ivo Cavoretto $^{a}$ Anna Seidenari $^{\mathrm{b}}$ Silvia Amodeo $^{\mathrm{b}}$ \\ Anna Nunzia Della Gatta ${ }^{b}$ Roberta Nale ${ }^{a}$ Yasmin Sara Ismail ${ }^{b}$ \\ Massimo Candiani ${ }^{a}$ Antonio Farina ${ }^{b}$ \\ aGynecology and Obstetrics Department, I.R.C.C.S. San Raffaele Hospital, University Vita-Salute, Milan, Italy; \\ ${ }^{b}$ Division of Obstetrics and Prenatal Medicine, Department of Medicine and Surgery (DIMEC), Sant'Orsola-Malpighi \\ Hospital, University of Bologna, Bologna, Italy
}

\section{Keywords \\ International Federation of Gynecology and Obstetrics 2015 cardiotocography classification · Feto-neonatal pH . Logistic regression · Risk estimation cardiotocography . Intrapartum fetal monitoring $\cdot$ Fetal surveillance $\cdot$ Birth asphyxia - Childbirth}

\begin{abstract}
Introduction: Intrapartum cardiotocography (CTG) was used for several decades to detect a stressed fetus so that delivery can be expedited to prevent birth asphyxia. The main aim of the study was to calculate the risk of neonatal acidemia ( $\mathrm{pH} \leq 7.10)$ according to duration of the 2 nd stage of labor and occurrence of the International Federation of Gynecology and Obstetrics (FIGO) 2015 CTG classification parameters. Materials and Methods: This was a retrospective case-control study on 552 pregnancies receiving continuous CTG monitoring in labor and immediate hemogasanalysis at birth. Cases with umbilical artery (UA) pH $\leq 7.10$ and controls with $\mathrm{UA} \mathrm{pH} \geq 7.10$ were matched for parity and gestational age at delivery, with ratio 1:5. Logistic regression analysis, adjusted for the expected risk in the general population, was used to calculate the baseline risk of $\mathrm{UA} \mathrm{pH} \leq 7.10$
\end{abstract}

in the absence of any CTG pathological feature and those associated with pathological CTG patterns occurring in the 2nd stage according to FIGO 2015. Results: Seventy-three cases and 387 controls reached 2 nd stage and were included in the analysis. For those reaching 2 nd stage, the mean adjusted risk of acidemia associated with nonpathological CTG was $1.6 \%$. Stratification of risk according to duration of the 2nd stage yielded risks of neonatal acidemia of 1.23, 2.08, 5.81 , and $15.22 \%$ at $30,60,120$, and 180 min, respectively. Bradycardia $>10$ min was associated with risk of neonatal acidemia of 9.9 and $15.8 \%$ for 2 nd-stage durations of 30 and 60 min, respectively. Risks associated with 1 prolonged deceleration $>5$ min were $6.80,11.08,27.0$, and $51.0 \%$ at 30,60 , 120 , and $180 \mathrm{~min}$, respectively. Repetitive late or prolonged decelerations $>30$ min were associated with risk of neonatal acidemia of $2.43,4.14,11.17$, and $26.45 \%$ at $30,60,120$, and $180 \mathrm{~min}$, respectively. Conclusion: The risk of neonatal acidemia is directly proportional to duration of the 2nd stage, irrespective of the presence of CTG abnormalities, increasing 12 -fold (1.2-15.3\%) from 30 to 180 min. Occurrence of FIGO 2015 pathological CTG patterns showed a decreasing impact from bradycardia $>10 \mathrm{~min}$ to decelerations $>5 \mathrm{~min}$, recurrent later or prolonged decelerations $>30 \mathrm{~min}$, and nonpathological CTG. karger@karger.com www.karger.com/fdt

(c) 2021 S. Karger AG, Basel

Karger!
Antonio Farina

Division of Obstetrics and Prenatal Medicine

Department of Medicine and Surgery (DIMEC), ant'Orsola-Malpighi Hospital

University of Bologna, Via Massarenti 13, IT-40138 Bologna (Italy)

antonio.farina@unibo.it 


\section{Introduction}

\section{Background}

Intrapartum cardiotocography (CTG) is the most common tool used in obstetrics to assess fetal well-being during labor and identify fetuses with developing hypoxia so that timely intervention can be undertaken in order to reduce the likelihood of neonatal encephalopathy [13]. The impact of intrapartum electronic fetal monitoring on neonatal outcomes continues to be controversial [1]. Despite the lack of solid evidences showing benefits of continuous or intermittent CTG monitoring (particularly in low-risk pregnancies), this approach became frequently the standard of care determining higher rates of caesarean sections and operative deliveries without significative reduction in cerebral palsy and infant morbidity [4-6].

A major limitation of CTG analysis is the lack of a reliable and patient-specific risk quantification since, at the present, only 3 basic levels have been reported (i.e., normal, suspicious, or pathological) [7]. This limitation is due to lack of adjustment of CTG traces' interpretation for intrapartum risk factors such as duration of labor and occurrence and duration of CTG abnormalities. Additional limitations reported with clinical use of CTG are related to the poor interobserver agreement in tracing interpretation, as well as to low positive predictive value for fetal acidosis (sensitivity 75-95\% and specificity 65-85\%), strengthening the concept that research is urgently needed on the topic of prediction of birth asphyxia $[4,5,8]$.

In the last 40 years, the International Federation of Gynecology and Obstetrics (FIGO), the American College of Obstetricians and Gynecologists (ACOG), and the United Kingdom National Institute for Health and Care Excellence (NICE) published several guidelines that focused on identifying fetal heart rate (FHR) tracings indicative of severe metabolic acidosis leading to brain injury $[7,9,10]$. It was shown that FIGO methodology presented higher sensitivity, but lower specificity, for the detection of neonatal acidemia, as compared to that proposed by ACOG or NICE [11]. However, no attempt was noted to quantify the risk of neonatal acidemia associated with each pathological feature and it is also unknown how quickly fetal oxygenation deteriorates in presence of a pathological CTG pattern.

\section{Objectives}

The primary aim of this study was to calculate the risk of neonatal acidemia $(\mathrm{pH} \leq 7.10)$ according to duration of the 2nd stage of labor and occurrence of the FIGO 2015
CTG classification parameters [7]. The secondary aim of this study was to assess correlation between duration of CTG abnormalities and neonatal umbilical artery (UA) $\mathrm{pH}$ reduction.

\section{Materials and Methods}

\section{Study Design and Setting}

This was a retrospective study assessing the risk of acidemia at birth $(\mathrm{pH} \leq 7.10)$, carried out on women who delivered at the Division of Prenatal Medicine, Department of Medical Science (DIMEC), University of Bologna, and Department of Obstetrics, San Raffaele Hospital, Vita-Salute University (Milan), between January 2019 and October 2019. The study was approved by the Local Ethics Committee of the Sant'Orsola Hospital and conducted in accordance with the ethical standards for human research established by the Declaration of Helsinki. The Ethics Committee also waived the authors from obtaining an informed consent because of the retrospective and anonymous data collection. The Ethics Committee code is DePreCar 805/2019. The study was presented according to the Strengthening the Reporting of Observational Studies in Epidemiology (STROBE) statement guidelines for reporting observational studies [12].

\section{Participants}

Ninety-two nonconsecutive pregnant women with a neonatal $\mathrm{pH}$ of $\leq 7.10$ (cases) as well as a group of 460 controls with a neonatal $\mathrm{pH}$ of $>7.10$ (ratio 1:5) were enrolled. In order to minimize selection bias, all consecutive cases fulfilling our criterion of neonatal acidemia in the study period were recruited. Retrospective selection of controls was carried out within the same day of each case with neonatal acidemia. Cases and controls were matched for gestational age (GA) at delivery and parity. Matching of randomly selected controls was achieved with inclusion of the first nonacidemic birth $(\mathrm{pH}>7.10)$, with concordant GA at delivery ( $<2$ days difference) and identical parity, following each case.

Inclusion criteria were singleton pregnancies at $38+0$ to $41+3$ gestational weeks and presence of determined quality requirements of CTG tracings including description of all CTG pathological features of FIGO 2015, which are described below. Exclusion criteria were nonvertex presentation, trial of labor after cesarean delivery, high-risk pregnancy (multiple gestation, pre-eclampsia, gestational hypertension, gestational diabetes mellitus, and intrauterine growth restriction), known fetal anomalies, intrauterine fetal demise, relevant placental abnormalities (including placenta previa or suspicion of morbidly adherence), significant maternal pathological conditions (including pregestational diabetes mellitus, nephropathy, chronic hypertension, and cardiac diseases), cesarean delivery for failure of induction or pathologic CTG at the first stage of labor, and prolonged 2nd stage of labor (see below). Fulfillment of inclusion criteria determined consideration for statistical analysis purpose, whereas presence of any of the exclusion criteria determined elimination from final analysis (Fig. 1, STROBE flowchart).

\section{Variables}

All patients recruited in the study received continuous CTG monitoring in labor and immediate funicular hemogasanalysis at birth. The 2 nd stage of labor starting point was defined at $10 \mathrm{~cm}$ of 
Fig. 1. STROBE flowchart of study recruitment. CTG, cardiotocography.

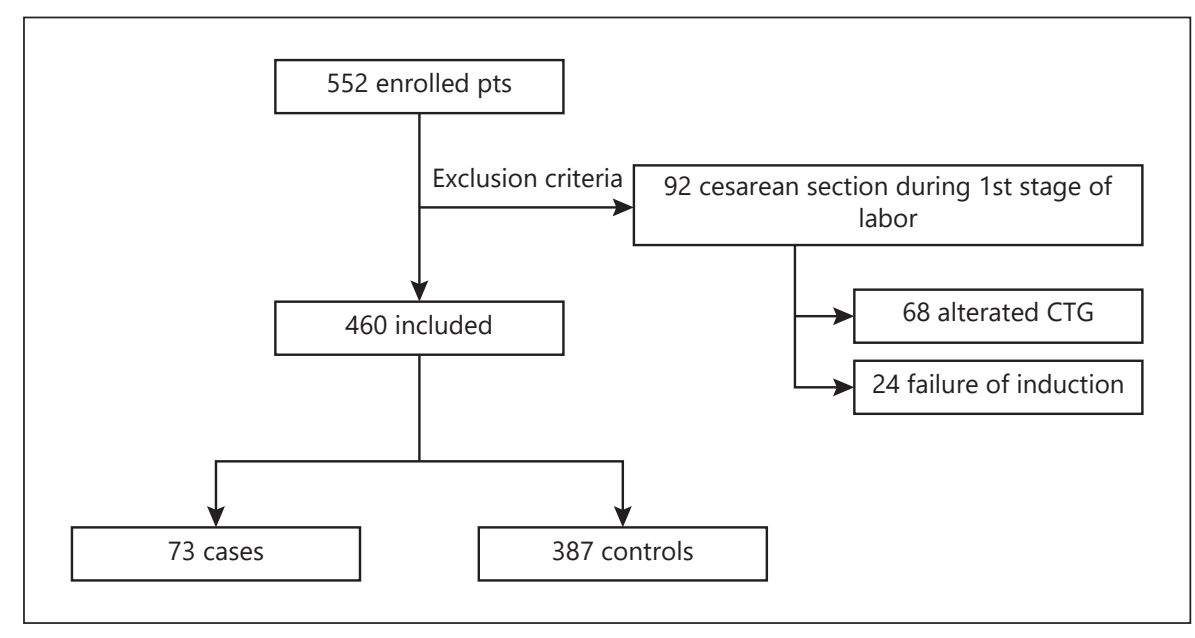

cervical dilatation or when the urge to bear down was present [13]. We defined neonatal acidemia when the UA pH was $\leq 7.10$ (outcome of the study). We defined prolonged 2nd stage of labor (failure to progress) as follows: in nulliparous, no progression after $3 \mathrm{~h}$ with regional anesthesia or $2 \mathrm{~h}$ without; in multiparous, no progression after $2 \mathrm{~h}$ with regional anesthesia or $1 \mathrm{~h}$ without [14, 15]. Failure of induction of labor was defined when active phase was not reached after $15 \mathrm{~h}$ of oxytocin started and rupture of membranes occurred [16]. The CTG traces were stored electronically for offline analysis at $1-\mathrm{cm} / \mathrm{min}$ paper speed. Since this was a retrospective study, information about the outcome (metabolic acidemia) was not blinded; however, no other clinical information was revealed.

\section{Data Sources and CTG Assessment}

Immediately after clamping the umbilical cord, funicular blood was aspirated from the UA with a $2-\mathrm{mL}$ syringe. Blood gas analysis was performed within $5 \mathrm{~min}$ from birth, using an automated blood gas analyzer located inside the labor ward (Bayer RAPIDLAB 865; Diamond Diagnostics, Holliston, MA, USA). Measurement of UA $\mathrm{pH}$ was recorded into a dedicated database including all patients' characteristics, duration of the 2 nd stage of labor, and presence/ duration of CTG pathological features according to FIGO 2015.

CTG was performed with fetal monitors (Avalon FM30; Philips Healthcare, Netherlands) equipped with ultrasound transducers applied on the abdominal surface or scalp transducers applied on the fetal presenting part, when external monitoring resulted inadequate due to signal loss. Simultaneous maternal heart rate was recorded in all cases in order to exclude wrong signal sampling.

The CTG traces and relative FHR patterns were assessed and classified post hoc in consensus by 2 experienced physicians (A.D.G. and R.N.) according to the FIGO 2015 CTG classification criteria published in the consensus guidelines [7]. In case of disagreement in the CTG interpretation, a different senior expert (P.C. and A.F.) settled. CTG pathological features of FIGO 2015 included were as follows: bradycardia $(\mathrm{BD})>10 \mathrm{~min}$, abnormal variability, sinusoidal pattern, repetitive late or prolonged decelerations $>30$ or $>20 \mathrm{~min}$ if reduced variability (repetitive late or prolonged deceleration [RLPD]), and single prolonged deceleration $>5$ min with FHR maintained at $<80$ beats per min (bpm) and reduced variability within the deceleration (prolonged deceleration [PD] $>5$ minutes; FHR maintained at $<80 \mathrm{bpm}$ and reduced variability within the deceleration). Normal variability was diagnosed in presence of a bandwidth amplitude of 5-25 bpm. Reduced variability was defined as a bandwidth amplitude below 5 bpm for $>50 \mathrm{~min}$ in baseline segments or for $>3$ min during decelerations and increased variability with bandwidth value exceeding $25 \mathrm{bpm}$ lasting $>30 \mathrm{~min}$. Sinusoidal pattern was defined as smooth, undulating signal, resembling a sine wave, with an amplitude of $5-15 \mathrm{bpm}$ and frequency of $3-5$ cycles per min, lasting $>30 \mathrm{~min}$ with absent accelerations. BD was defined when the FHR was below $100 \mathrm{bpm}$ for a duration of $>10 \mathrm{~min}$. RLPD was defined when duration exceeded $30 \mathrm{~min}$, or $20 \mathrm{~min}$ when associated with reduced short-term variability $(<5 \mathrm{bpm})$. Decelerations were defined repetitive when associated with $>50 \%$ of uterine contractions [7].

\section{Bias}

Risk of bias was assessed and minimized with random selection of study participants, within the defined matching protocol (GA and parity), homogeneous use of analytic procedures to assess neonatal hemogasanalysis, and standardization of reading of CTG traces according to FIGO criteria, published in 2015. Differences in the nature of patient's characteristics were deeply taken into consideration to minimize risk of bias due to the effect of significant confounders, mediators, or moderators. However, some random effect due to patient-specific characteristic could not be intercepted by our selection.

\section{Statistical Methods}

Descriptive analysis was performed by routine tests including Student's $t$ test, Mann-Whitney $\mathrm{U}$ test, and $\mathrm{Z}$ test for comparison of 2 different proportions. K-Cohen statistics was used to evaluate the agreement between 2 raters located in each of the 2 centers in which the study took place.

The risk of $\mathrm{pH} \leq 7.10$ at delivery has been calculated with the following strategies:

1. The risk of $\mathrm{pH} \leq 7.10$ of our population has been adjusted following the method reported by Collett [17] that calculates the "adjustment fraction" using risk that was reported in the medical literature [18]. 
Table 1. Epidemiological and clinical characteristics of the study population according to the study group

\begin{tabular}{|c|c|c|c|c|c|c|}
\hline & $\mathrm{pH}>7.1(460)$ & $\mathrm{pH} \leq 7.1(92)$ & $p$ value* & $\mathrm{pH}>7.1(387)$ & $\mathrm{pH} \leq 7.1(73)$ & $p$ value* \\
\hline Maternal BMI prepregnancy & $22.48(3.78)$ & $23.79(4.72)$ & 0.004 & $22.46(3.72)$ & $23.78(4.68)$ & 0.014 \\
\hline Maternal BMI at delivery & $26.8(3.85)$ & $28.2(5.53)$ & 0.004 & $26.73(3.79)$ & $28.02(5.52)$ & 0.004 \\
\hline Gestational age at delivery, weeks + days & $39+6(1+1)$ & $39+6(1+1)$ & 0.960 & $39+6(1+1)$ & $39+6(1+1)$ & 0.655 \\
\hline Primiparous & $142(30.9)$ & $23(25.0)$ & 0.263 & $135(34.9)$ & $19(26.0)$ & 0.142 \\
\hline Multiparous & $22(4.8)$ & $3(3.3)$ & 0.522 & $22(5.7)$ & $2(2.8)$ & 0.298 \\
\hline \multicolumn{7}{|l|}{ Racial origin, $n(\%)$} \\
\hline Caucasian & $412(89.5)$ & $79(85.9)$ & 0.303 & $345(89.2)$ & $62(84.9)$ & 0.303 \\
\hline Afro-Caribbean & $15(3.3)$ & $1(1.1)$ & 0.258 & $11(2.8)$ & $1(1.4)$ & 0.472 \\
\hline APGAR 1 & $9(0.7)$ & $7(2.2)$ & $<0.001$ & $9(0.6)$ & $8(1.9)$ & $<0.001$ \\
\hline APGAR 5 & $10(0.4)$ & $9(1.0)$ & $<0.001$ & $10(0.3)$ & $9(0.8)$ & $<0.001$ \\
\hline Neonatal weight, $g$ & $3,320(423)$ & $3,349(448)$ & 0.569 & $3,343(424)$ & $3,362(474)$ & 0.753 \\
\hline
\end{tabular}

* Student's $t$ test or Mann-Whitney U test or test for comparison of 2 proportions was used. Continuous data are expressed as mean $(\mathrm{SD})$ and categorical data as frequency (percentage).

Table 2. Abnormal CTG patterns observed in women that did not experience the 2 nd stage of labor $(N=92)$

\begin{tabular}{rllll}
\hline $\begin{array}{l}\text { Cases, } \\
n\end{array}$ & Bradycardia & $\begin{array}{l}\text { Variability } \\
>25 \mathrm{~b} / \mathrm{min}\end{array}$ & $\begin{array}{l}\text { Repetitive* } \\
\text { late or prolonged } \\
\text { decelerations }\end{array}$ & $\begin{array}{l}\text { One prolonged } \\
\text { deceleration } \\
>5 \text { min }\end{array}$ \\
\hline 24 & No & No & No & No \\
35 & No & No & Yes & No \\
12 & No & No & No & Yes \\
8 & Yes & No & No & No \\
10 & No & No & Yes & Yes \\
2 & Yes & No & No & Yes \\
1 & Yes & Yes & No & No \\
\hline $\mathbf{9 2}$ & $\mathbf{1 1}$ & $\mathbf{1}$ & $\mathbf{4 5}$ & $\mathbf{2 4}$ \\
\hline
\end{tabular}

CTG, cardiotocography. * Decelerations are repetitive when associated with $>50 \%$ contractions.

2. Calibration of the logistic equation having the 2 nd stage of labor as independent variable: the adjustment fraction $\left(\beta_{\text {adj }}\right)$ after natural log conversion was added to the constant $\left(\beta_{0}\right)$ of the univariable logistic model obtained from our results. $\mathrm{pH}$ values (dependent variable) were dichotomized in $\leq 7.10$ and $>7.10$ and stratified according to the duration of 2nd stage of labor which constitutes the independent variable. Given these as- sumptions, the mean risk of neonatal $\mathrm{UA} \mathrm{pH} \leq 7.10$ calculated from the logistic regression must amount to that taken from the literature.

3. Calculation of the baseline risk: in order to obtain the risk of $\mathrm{pH} \leq 7.10$ in absence of any CTG features suggestive of a pathological CTG according to FIGO 2015, each CTG feature was dichotomized and added to the regression model. Baseline risk $\left(\beta_{\text {adj }}+\beta_{0}\right)$ was that obtained when all the features were absent.

4. Multivariable estimation of the risk of $\mathrm{pH} \leq 7.10$ generated adding to the duration of the 2 nd stage of labor and the presence of each available feature of pathological CTG according to FIGO 2015 classification.

5. Finally, a simple linear regression was used to estimate the rate of $\mathrm{pH}$ reduction as a function of the duration of the FIGO 2015 pathological features.

\section{Results}

Figure 1 shows the STROBE flowchart of study recruitment. Table 1 reports the demographic and clinical characteristics available for the whole series of data and for those who experienced the 2nd stage. As shown, the variables that were statistically different between cases and controls in the whole series of data remained different in the population that experienced only the 1st stage or both 1 st and 2 nd stages of labor. 
Table 3. Number and cumulative duration of pathological features per patient

\begin{tabular}{|c|c|c|c|c|c|c|}
\hline FIGO 2015 variable & $\begin{array}{l}\text { Cumulative } \\
\text { duration } \\
\text { of features at } \\
\text { 1st stage, min }\end{array}$ & $\begin{array}{l}\text { Cumulative } \\
\text { duration } \\
\text { of features at } \\
\text { 2nd stage, min }\end{array}$ & $\begin{array}{l}\text { Episodes of } \\
\text { 1st stage, } n\end{array}$ & $\begin{array}{l}\text { Episodes of } \\
\text { 2nd stage, } n\end{array}$ & $\begin{array}{l}\text { Patients with } \\
\text { features at } \\
\text { 1st stage, } n\end{array}$ & $\begin{array}{l}\text { Patients with } \\
\text { features at } \\
\text { 2nd stage, } n\end{array}$ \\
\hline Bradycardia $>10 \mathrm{~min}$ & - & $13(10-25)$ & - & 1 & - & 18 \\
\hline RLPD* & $81(31-253)$ & $50(32-86)$ & $\begin{array}{l}1 \\
2 \\
3 \\
4 \\
5\end{array}$ & $\begin{array}{l}1 \\
2 \\
- \\
- \\
-\end{array}$ & $\begin{array}{r}44 \\
33 \\
13 \\
4 \\
1\end{array}$ & $\begin{array}{r}22 \\
6 \\
- \\
- \\
-\end{array}$ \\
\hline One deceleration $>5 \mathrm{~min}$ & - & $6(5-20)$ & $\begin{array}{l}- \\
- \\
- \\
-\end{array}$ & $\begin{array}{l}1 \\
2 \\
3 \\
4\end{array}$ & $\begin{array}{l}- \\
- \\
- \\
-\end{array}$ & $\begin{array}{r}35 \\
1 \\
1 \\
1\end{array}$ \\
\hline
\end{tabular}

Cumulative duration per patient $(\mathrm{min})$ : number of episodes of each feature stratified for number of patients occurring in the 1st and 2nd stages of labor. Data are expressed as median (min-max). Each feature is analyzed with dichotomization (present/absent). FIGO, International Federation of Gynecology and Obstetrics; RLPD, repetitive late or prolonged deceleration. * RLPD: repetitive (when associated with $>50 \%$ contractions), prolonged, or late decelerations $>30 \mathrm{~min}$.

\section{Main Results}

Interobserver agreement (A.D.G. working in Bologna and R.N. working in Milan) is reported in online suppl. Table 1; for all online suppl. material, see http://www. karger.com/doi/10.1159/000512658. As expected, the lower percentage of agreement was found for RLPD. PD was associated with the higher degree of agreement.

The quoted sensitivity and specificity according with FIGO 2105 were 72.8 and $61.5 \%$, respectively. It must be noted that given the statistical design, we did not expect any modification of the sensitivity and specificity using such multivariable approach since we did not introduce any extra and/or new variable. Ninety-two out of 552 cases did not experience the 2 nd stage of labor and had cesarean sections during the 1st stage of labor: $68(74 \%)$ due to pathological CTG features and $24(26 \%)$ due to failure of induction (Table 2). Out of this subgroup, we observed 15 cases $(16.3 \%)$ of $\mathrm{pH} \leq 7.10$ at delivery.

Seventy-three cases and 387 controls reached the 2 nd stage of labor and were included to the analysis (460 pregnancies; ratio 1:5.3; quite similar to the original match $1: 5)$. The rate of delivery in the overall group of $387 \mathrm{pa}-$ tients was calculated at fixed time points of $\leq 60, \leq 120$, and $\leq 180$ min according with mode of delivery. For spontaneous delivery, the rates were 82,95 , and $100 \%$. For vacuum delivery, the rates were 67,93 , and $100 \%$. For cesarean section, the rates were 36,43 , and $100 \%$. The rates of cesarean section and vacuum delivery were 5.5 versus $1.8 \%$ and 19.2 versus $18.3 \%$ in 73 cases and 387 controls, respectively.

Table 3 reports the number and cumulative duration of pathological features per patient occurring in the 460 cases who experienced the 2nd stage of labor in their 1st and 2 nd stages of labor. As shown, during the 1st stage of labor, only RLPD was present with a mean cumulative duration per patient of $81(31-253)$ min and up to 5 times per patient, but it was decided to go on with the labor.

RLPD in the 2nd stage occurred up to 2 times per patient and for an average cumulative duration per patient of $50(32-86) \mathrm{min}$. BP and PD occurred, as expected, only in the 2 nd stage of labor with a mean cumulative duration per patient of $13(10-25)$ and $6(5-20) \mathrm{min}$.

Assuming a risk of $3.4 \%$ of $\mathrm{pH} \leq 7.10$ (irrespective of the CTG and the duration of labor), for those patients reaching the 2nd stage of labor, the mean adjusted risk of acidemia associated with a nonpathological CTG (weighted for number of cases) was $1.6 \%$. Using duration of the 2nd stage of labor as an extra risk factor, we observed a risk of $\mathrm{pH} \leq 7.10$ of $1.23,2.08,5.81$, and $15.22 \%$ at 30,60 , 120 , and $180 \mathrm{~min}$, respectively (Table 4; Fig. 2).

The addition of the FIGO CTG pathological features to the statistical model yielded significant association with a $\mathrm{pH}$ of $\leq 7.10$ for $\mathrm{BD}, \mathrm{PD}$, and RLPD. In fact, as shown in Table 4, BD was associated with risk of neonatal 
Fig. 2. Baseline risk (nonpathological CTG) of neonatal acidemia and that of each significant feature stratified according to duration of the 2nd stage of labor. CTG, cardiotocography; RLPD, repetitive late or prolonged deceleration.

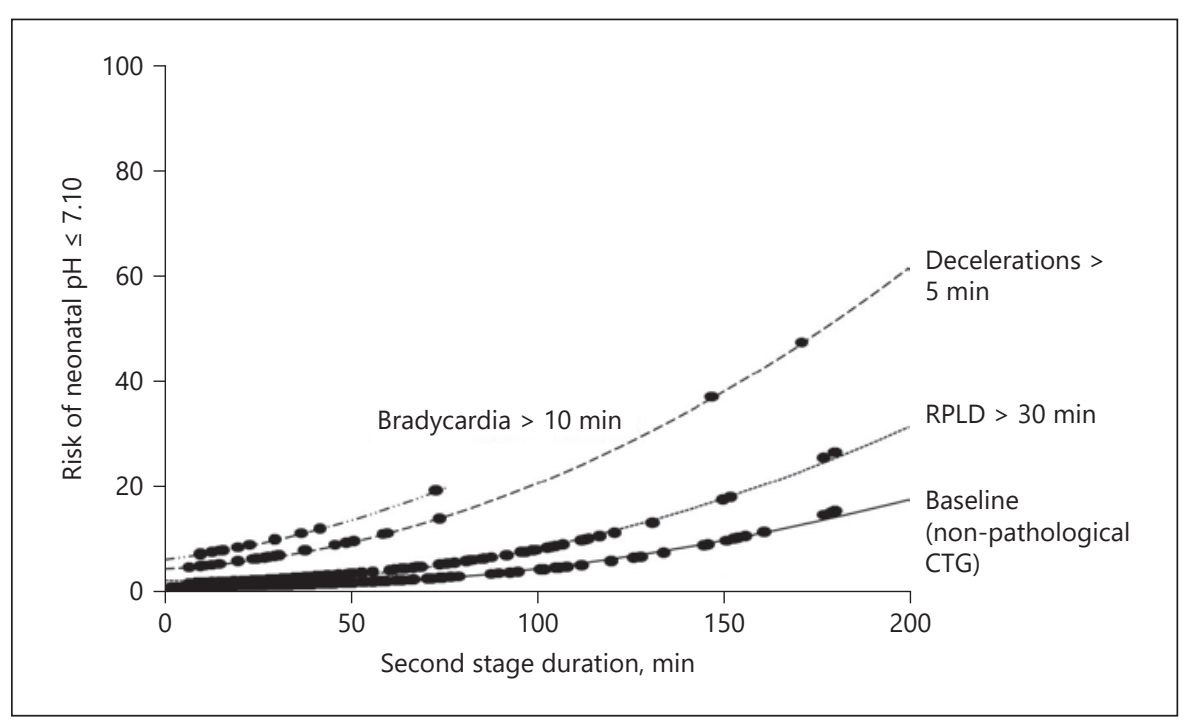

Table 4. Risk of neonatal acidemia and latency from onset of CTG pathological features to birth

\begin{tabular}{|c|c|c|c|c|c|c|c|c|}
\hline \multirow[b]{2}{*}{$\begin{array}{l}\text { 2nd-stage duration, min } \\
\text { FIGO } 2015 \text { classification }\end{array}$} & \multicolumn{4}{|c|}{ Mean risk (SEM) of neonatal acidemia, \% } & \multicolumn{4}{|c|}{ Time interval, from onset of the CTG feature to birth, min } \\
\hline & 30 & 60 & 120 & 180 & $\leq 30$ & $\leq 60$ & $\leq 120$ & $\leq 180$ \\
\hline \multicolumn{9}{|l|}{ Pathological features } \\
\hline Bradycardia $>10 \mathrm{~min}$ & $9.90(0.12)$ & $15.80(0.13)$ & - & - & $11.5(10-21)$ & $15(12-55)$ & - & - \\
\hline RLPD* & $2.43(0.01)$ & $4.14(0.03)$ & $11.17(1.39)$ & $26.45(0.99)$ & $30(17-30)$ & $51(30-70)$ & $77(30-120)$ & $86(42-150)$ \\
\hline
\end{tabular}

Risk (\%) of neonatal acidemia ( $\mathrm{pH} \leq 7.10$ ) according to duration of the 2 nd stage of labor and time interval from onset of the FIGO 2015 CTG pathological features to birth according to 2 nd-stage duration $(n=460)$. Data are expressed as mean (min-max). CTG, cardiotocography; SEM, standard error of mean; FIGO, International Federation of Gynecology and Obstetrics; RLPD, repetitive late or prolonged deceleration. * RLPD: repetitive (when associated with $>50 \%$ contractions), prolonged, or late decelerations $>30 \mathrm{~min}$.

acidemia of 9.9 and $15.8 \%$ for 2 nd-stage durations of 30 and $60 \mathrm{~min}$, respectively (mean time [min] from BD onset to delivery: 16; range 10-55). No cases of BD occurred in patients with a 2 nd stage of $>60 \mathrm{~min}$. RLPD was associated with risk of neonatal acidemia of $2.43,4.14,11.17$, and $26.45 \%$ at $30,60,120$, and $180 \mathrm{~min}$, respectively (mean time [min] from RLPD onset to delivery: 61; range 24-139). Risks associated with PD were 6.80, 11.08, 27.0, and $51.0 \%$ at $30,60,120$, and $180 \mathrm{~min}$, respectively (mean time [min] from PD onset to delivery: 64; range 5-180).

Figure 2 reports the baseline risk (nonpathological CTG) and that of each significant feature stratified according to duration of the 2nd stage of labor. Figure 3 proposes a (flowchart of intervention) clinical risk assess- ment algorithm aimed at keeping the risk of neonatal $\mathrm{pH}$ $\leq 7.10$ below $3.4 \%$. We identified 3 risk levels of $\mathrm{pH} \leq 7.10$, based on a probabilistic strategy. The risk was considered low when inferior than the expected for the general population (3.4\%), high when $>15 \%$ (maximum risk observed at $180 \mathrm{~min}$ for a woman with a nonpathological CTG trace), and intermediate when ranging between the 2 extremes (from 3.4 to $15 \%$ ).

Finally, linear correlations were also found between both the duration of $\mathrm{BD}$ or duration of $\mathrm{PD}$ and reduction of UA pH ( $p$ value $<0.001$ for both). Each additional minute of BD exceeding $10 \mathrm{~min}$ and each 2 -min PD exceeding 5 min were associated with a $\mathrm{pH}$ reduction of 0.1 units. RLPD duration did not correlate with the $\mathrm{pH}$ reduction. 


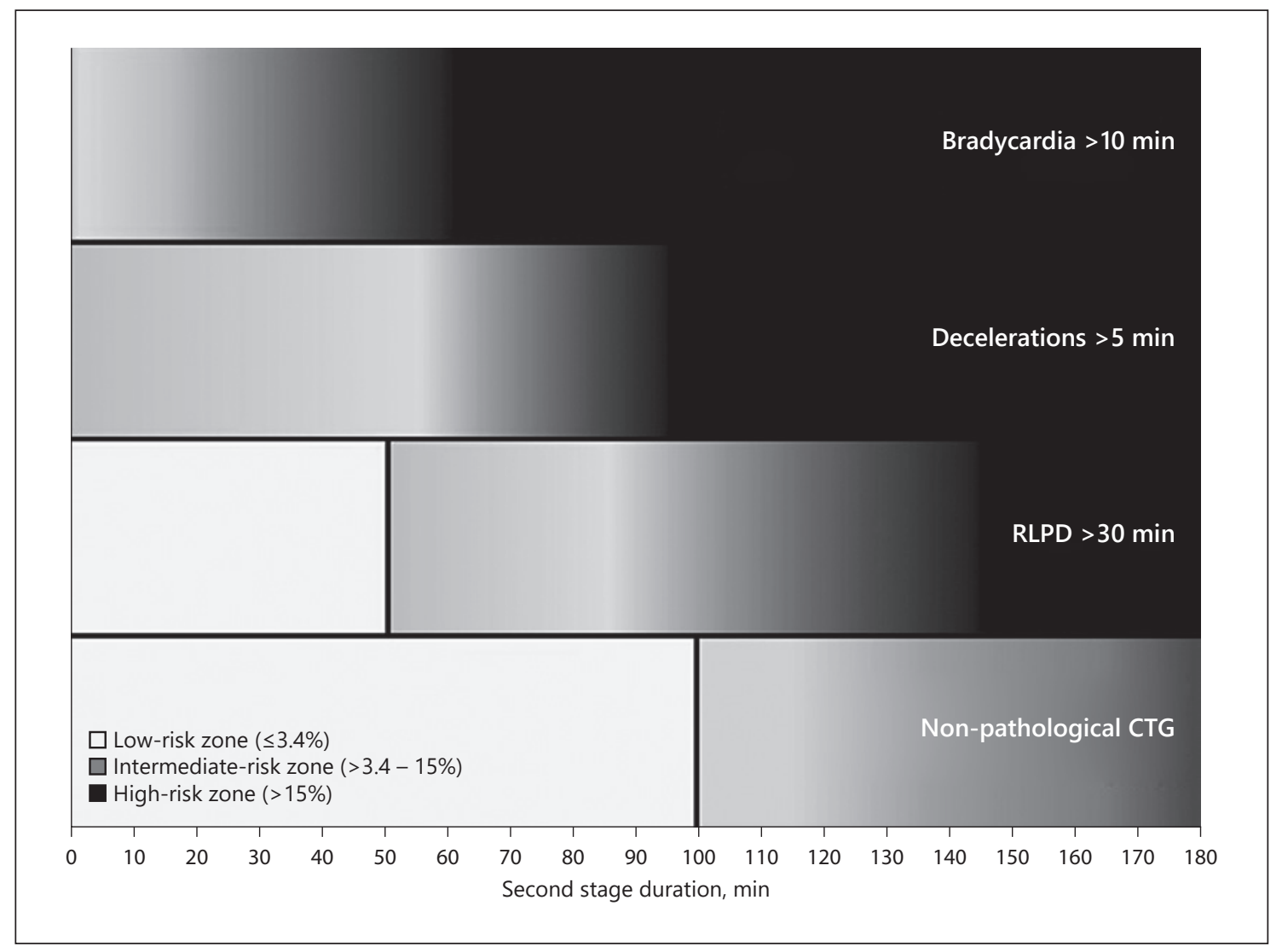

Fig. 3. Clinical risk assessment algorithm. CTG, cardiotocography; RLPD, repetitive late or prolonged deceleration.

\section{Discussion}

\section{Summary of Key Findings}

This study highlights some novel findings. First, the risk of neonatal acidemia is directly proportional to duration of the 2nd stage of labor in presence of nonpathological CTG or with occurrence of FIGO pathological patterns. Second, in the presence of $\mathrm{BD}$, the risk is always increased above $3.4 \%$ and becomes very high $(>15 \%)$ at $60 \mathrm{~min}$ of 2nd-stage duration. Third, in the presence of $\mathrm{PD}$, the risk is always increased above $3.4 \%$ and becomes very high at $100 \mathrm{~min}$ from onset of the 2 nd stage. Fourth, in the presence of RLPD, the risk increases above $3.4 \%$ at $50 \mathrm{~min}$ and becomes very high at $150 \mathrm{~min}$ from onset of the 2 nd stage. Finally, since risk calculation was related to the duration of 2 nd stage of labor, our study emphasizes the need for a timely diagnosis of beginning of 2 nd stage. In view of these considerations, the present algorithm leads to individualization of risk at qualitative and quantitative assessment of CTG, with potential interest at the bedside for clinical decision purposes.

FIGO 2015 Abnormal CTG Features and Risk of Neonatal Acidemia

\section{Interpretation}

This is the first paper to our knowledge that evaluates the FIGO 2015 criteria for CTG assessment from a different point of view, based on the risk quantification derived from both duration of the 2nd stage of labor and from each of the features observed in each trace. We used as adjustment factor the risk for the general population found in the medical literature, but it must be focused that the adjustment and the relative proper definition of the high-risk cases may vary with the different populations and/or with the specific clinical protocols used in different countries.

$\mathrm{BD}$ with duration $>10$ min was the most important prognostic factor and increased the baseline risk of about 9-fold, followed by the presence of PD that was associated with a risk of about 6-fold and RLPD which was less dangerous for neonatal $\mathrm{pH}$, associated with a risk increase of about 2 -fold. Given the statistical assumption used in the analysis, the risk increase associated with each CTG pathological feature was constant at any time of labor. Therefore, for a given feature, the same magnitude of risk in- 
crease (or odds ratio) occurred constantly, regardless of time appearance. However, the absolute risk estimation was influenced by both CTG feature occurrence and duration of labor, being higher for labors of longer durations.

We also estimated as secondary outcome the $\mathrm{pH}$ reduction for each minute of appearance of BP and PD. However, this result, to be prospectively useful, must take into account the fetal acid-base status at the time of appearance of the feature that remains a hard challenge to be evaluated a priori. Therefore, future studies on this topic may need to consider fetal scalp blood analysis when developing algorithms of CTG interpretation.

\section{Strengths and Limitations}

The traditional risk assessment from a case-control study was adjusted for the expected risk of a low $\mathrm{pH}$ of $\leq 7.10$ in the general population. We also calculated the $\mathrm{pH}$ deterioration for each minute of duration of the features associated with a low neonatal $\mathrm{pH}$. We believe that this methodological approach may lead to future studies with a high potential of generalization, in order to create individual patient-specific risks in labor within a precision medicine approach. A customized risk assessment could provide clinicians room for improvement of clinical practice with specific and timely monitoring or interventions.

Several limitations must be reported. (i) The CTG interpretation with intra- and interobserver variation is a weak point of every paper on this topic, and we acknowledge possible errors. To minimize this component, we performed an analysis of interobserver variation (online suppl. Table 1). (ii) We assumed that the presence of each feature has a constant risk increase in the $\mathrm{pH}$ deterioration irrespective of the time of appearance during the labor. (iii) No proper correction for possible covariates on the low $\mathrm{pH}$ occurrence has been performed such as BMI and epidural analgesia given the relative low number of cases. However, this limitation does not affect the validity of our model since the study aim was to assess CTG abnormalities. Moreover, the effect of epidural was shown to be not significant by a previous meta-analysis [19]. (iv) Some important features such as sinusoidal pattern and variability $>25 \mathrm{bpm}$ were not present in our dataset, therefore precluding any possible risk estimation of these important features. (v) The time of intervention is different for a vacuum delivery and a cesarean delivery, and therefore, the $\mathrm{pH}$ is probably a bit lower in such group given the same CTG feature pattern. (vi) Since this is a retrospective study, we acknowledge that calculated posterior risk of neonatal acidemia could be overestimated.

\section{Conclusion}

In this paper, we put the basis for the quantification of the risk of neonatal acidemia according to the 2nd stage of labor duration and FIGO 2015 CTG features. In addition, we propose an algorithm to be used for defining the patient-specific risk of acidemia in real clinical settings. This method potentially provides evidence-based foundations for reproducible decisions in labor ward and timely interventions.

\section{Statement of Ethics}

This study was approved by the Local Ethics Committee of the Sant'Orsola Hospital and conducted in accordance with the ethical standards for human research established by the Declaration of Helsinki. The Ethics Committee code is DePreCar 805/2019. The Ethics Committee waived the authors from obtaining an informed consent because of the retrospective and anonymous data collection.

\section{Conflict of Interest Statement}

The authors report no conflicts of interest.

\section{Funding Sources}

The authors did not receive any funding.

\section{Author Contributions}

Paolo Ivo Cavoretto, Massimo Candiani, and Antonio Farina contributed to the study conception and design. Anna Seidenari, Silvia Amodeo, Anna Nunzia Della Gatta, Roberta Nale, and Yasmin Sara Ismail were involved in data collection. Anna Nunzia della Gatta and Roberta Nale evaluated the CTG. Paolo Ivo Cavoretto and Antonio Farina supervised CTG interpretation to resolve conflicts, performed data analysis, and wrote the paper. All authors contributed to editing of the manuscript. 


\section{References}

1 Nelson KB, Dambrosia JM, Ting TY, Grether $J K$. Uncertain value of electronic fetal monitoring in predicting cerebral palsy. N Engl J Med. 1996;334(10):613-8.

2 Clark SL, Hankins GD. Temporal and demographic trends in cerebral palsy: fact and fiction. Am J Obstet Gynecol. 2003;188(3):62833.

3 Macones GA, Hankins GD, Spong CY, Hauth J, Moore T. The 2008 National Institute of Child Health and Human Development workshop report on electronic fetal monitoring: update on definitions, interpretation, and research guidelines. Obstet Gynecol. 2008; 112(3):661-6.

4 Vintzileos AM, Nochimson DJ, Guzman ER, Knuppel RA, Lake M, Schifrin BS. Intrapartum electronic fetal heart rate monitoring versus intermittent auscultation: a meta-analysis. Obstet Gynecol. 1995;85(1):149-55.

5 Devane D, Lalor JG, Daly S, McGuire W, Cuthbert A, Smith V. Cardiotocography versus intermittent auscultation of fetal heart on admission to labour ward for assessment of fetal wellbeing. Cochrane Database Syst Rev. 2012;(2):CD005122.

6 Alfirevic Z, Devane D, Gyte GM, Cuthbert A. Continuous cardiotocography (CTG) as a form of electronic fetal monitoring (EFM) for fetal assessment during labour. Cochrane Database Syst Rev. 2017;2(2):CD006066.
7 Ayres-de-Campos D, Spong CY, Chandraharan E; FIGO Intrapartum Fetal Monitoring Expert Consensus Panel. FIGO consensus guidelines on intrapartum fetal monitoring: cardiotocography. Int $\mathrm{J}$ Gynaecol Obstet. 2015;13:13-24.

8 Schiermeier S, Pildner von Steinburg S, Thieme A, Reinhard J, Daumer M, Scholz M, et al. Sensitivity and specificity of intrapartum computerised FIGO criteria for cardiotocography and fetal scalp $\mathrm{pH}$ during labour: multicentre, observational study. BJOG. 2008 Nov;115(12):1557-63.

9 American College of Obstetricians and Gynecologists (ACOG).. Practice bulletin no. 116: management of intrapartum fetal heart rate tracings.. Obstet Gynecol. 2010;116(5):123240.

10 National Institute of Health and Care Excellence (NICE).. Intrapartum care for healthy woman and babies. CG190. [accessed 2012 Jan 6]. 2017. https://www.nice.org.uk/guidance/cg190.

11 Santo S, Ayres-de-Campos D, Costa-Santos C, Schnettler W, Ugwumadu A, Da Graça LM. Agreement and accuracy using the FIGO, ACOG and NICE cardiotocography interpretation guidelines. Acta Obstet Gynecol Scand. 2017;96(2):166-75.

12 von Elm E, Altman DG, Egger M, Pocock SJ Gøtzsche PC, Vandenbroucke JP. The Strengthening the Reporting of Observational Studies in Epidemiology (STROBE) statement: guidelines for reporting observational studies. J Clin Epidemiol. 2008;61(4):344-9.
13 Abalos E, Oladapo OT, Chamillard M, Díaz $\mathrm{V}$, Pasquale J, Bonet M, et al. Duration of spontaneous labour in "low-risk" women with "normal" perinatal outcomes: a systematic review. Eur J Obstet Gynecol Reprod Biol. 2018;223:123-32.

14 Holzmann M, Wretler S, Cnattingius S, Nordström L. Cardiotocography patterns and risk of intrapartum fetal acidemia. J Perinat Med. 2015;43(4):473-9.

15 Zipori Y, Grunwald O, Ginsberg Y, Beloosesky R, Weiner Z. The impact of extending the second stage of labor to prevent primary cesarean delivery on maternal and neonatal outcomes. Am J Obstet Gynecol. 2019; 220(2):191-e7.

16 Grobman WA, Bailit J, Lai Y, Reeddy UM, Wapner RJ, Varner MV, et al. Defining failed induction of labor. Am J Obstet Gynecol. 2018;218:122.e1-e8.

17 Collett D. Modelling binary data. New York, NY: Chapman and Hall/CRC; 2003.

18 Sabol BA, Caughey AB. Acidemia in neonates with a 5-minute Apgar score of 7 or greater: what are the outcomes? Am J Obstet Gynecol. 2016;215(4):486-6.

19 Reynolds F, Sharma SK, Seed PT. Analgesia in labour and fetal acid-base balance: a metaanalysis comparing epidural with systemic opioid analgesia. BJOG. 2002 Dec;109(12): 1344-53. 